\title{
Model System for a One-Dimensional Magnetic Photonic Crystal
}

\author{
S. Linden \\ Institut für Nanotechnologie, Forschungszentrum Karlsruhe in der Helmholtz-Gemeinschaft, D-76021 Karlsruhe, Germany \\ M. Decker and M. Wegener \\ Institut für Angewandte Physik and DFG-Center for Functional Nanostructures (CFN), Universität Karlsruhe (TH), \\ D-76131 Karlsruhe, Germany
}

(Received 17 February 2006; published 25 August 2006)

\begin{abstract}
We fabricate and characterize one-dimensional magnetic (rather than dielectric) photonic crystals for the first time. Our model system is a one-dimensional periodic lattice of gold-wire pairs. Each pair can be viewed as a magnetic coil with two slits and represents a "magnetic atom." Strong coupling between the resulting magnetic-dipole resonance and the Bragg resonance is accomplished by an adjacent dielectric slab waveguide, giving rise to an avoided crossing at near-infrared wavelengths. Our experimental findings are in excellent agreement with theory.
\end{abstract}

DOI: 10.1103/PhysRevLett.97.083902

PACS numbers: 42.70.Qs, 73.20.Mf

Maxwell's equations predict that both a periodic modulation of the electric permittivity $\epsilon$ (describing the electricdipole density) as well as a periodic modulation of the magnetic permeability $\mu$ (describing the magnetic-dipole density) can lead to Bragg scattering, Bloch waves, photonic band structures, and photonic band gaps. At microwave frequencies, magnetic materials with $\mu \neq 1$ are available [1]. At optical frequencies, however, the optical properties of periodic structures, i.e., photonic crystals [2], have been exclusively determined by the spatial modulation of $\epsilon$ so far. The magnetic permeability has played no role until now, since it is unity $(\mu=1)$ for all known natural substances in this spectral region. Metamaterials [3] composed of "artificial atoms" exhibiting a magneticdipole moment $(\mu \neq 1)$ at optical frequencies have become available only recently [4-9]. Naturally, the question arises whether it is possible to actually fabricate photonic crystals made from such "magnetic atoms."

What effects do we anticipate? Photonic crystals based on electric dipoles have addressed mostly dielectric constituent materials (e.g., silicon) which are off-resonant, i.e., for which the refractive index is approximately constant (frequency-independent) in the region of interest. No offresonant magnetic counterpart is known today. All magnetic atoms reported so far exhibit resonances. Thus, it is instructive to briefly recall the effect of resonances on dielectric photonic crystals, which has been discussed recently $[10,11]$. The authors find that the coupling of the dielectric resonance with the other resonance, the photonic mode or Bragg resonance, is closely analogous to two coupled harmonic oscillators and leads to an avoided crossing. In turn, the avoided crossing can be used as the fingerprint of the coupling of the two modes and, hence, as the fingerprint of a photonic crystal composed of a constituent dielectric material, which exhibits a resonance. Following this reasoning and our introduction, we expect an analogous avoided crossing for a magnetic photonic crystal, built from magnetic atoms exhibiting a resonance.
In this Letter, we study periodic arrays of magnetic atoms on top of a dielectric slab waveguide. These structures serve as a model system for one-dimensional (1D) magnetic photonic crystals [see Fig. 1(a)]. While our mag-

(a)

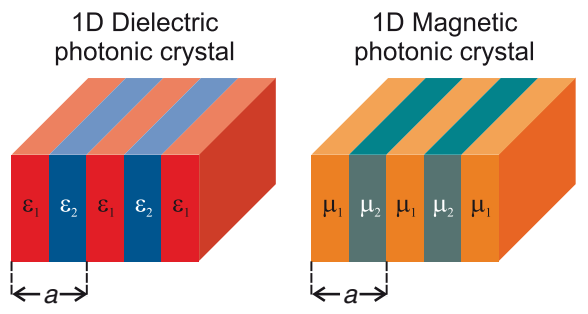

(b)

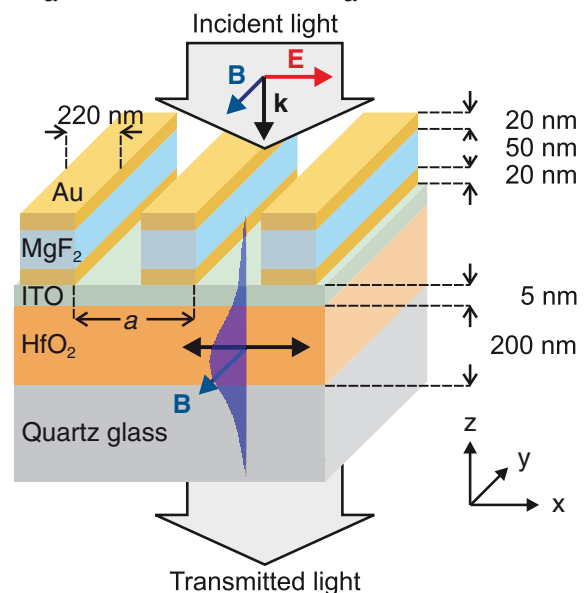

(c)

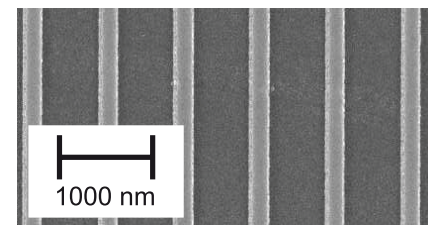

FIG. 1 (color). (a) Illustration of dielectric versus magnetic photonic crystals. (b) Scheme of our model system for a onedimensional magnetic photonic crystal (not to scale) composed of gold-wire pairs on top of a dielectric waveguide. (c) Top-view electron micrograph of a typical sample $(a=800 \mathrm{~nm})$. 
netic photonic crystals in slab geometry are obviously periodic along the $x$ direction [Fig. 1(b)], we can conveniently perform spectroscopy under normal incidence with respect to the substrate [see thick gray arrows in Fig. 1(b)]. This approach has frequently been used for spectroscopy of leaky modes of photonic crystal slabs [11-13] and is related to a usual grating coupler. In essence, the magnetic atoms in Fig. 1(b) are pairs of long gold wires (width $w=$ $220 \mathrm{~nm}$, height $h=20 \mathrm{~nm}$, length $l=100 \mu \mathrm{m}$ ), which are separated by a 50-nm-thick magnesium fluoride $\left(\mathrm{MgF}_{2}\right)$ spacer. For polarization of the incident light perpendicular to the wire pairs (TM polarization), the magnetic atoms exhibit two pronounced resonances in the spectral region of interest. These resonances correspond to the eigenmodes of the wire pair which result from coupling of the electric-dipole resonances of the two individual wires $[7,8,14]$. The long-wavelength resonance (magnetic resonance) is associated with the antisymmetric eigenmode. Here the electric fields (and, hence, the Ohmic currents) in the two wires point in opposite directions, and the magnetic field is concentrated in the $\mathrm{MgF}_{2}$ spacer oriented along the wires. This eigenmode supports a strong magnetic-dipole moment. In contrast, the shortwavelength resonance (electric resonance) stems from the excitation of the symmetric eigenmode. Since the electric fields in the wires are almost in phase in this case, the electric resonance has mainly electric-dipole character. For a polarization of the incident field parallel to the wires (TE polarization), the arrays act as diluted metals [15] and no resonances occur in the spectral region of interest. In the remainder of the Letter, we will mostly concentrate on the magnetic resonance and its interaction with photonic modes while investigating magnetic photonic crystals.

The dielectric slab waveguide utilized in our experiments is formed by a quartz glass substrate covered with $200 \mathrm{~nm}$ hafnium oxide $\left(\mathrm{HfO}_{2}\right)$ and $5 \mathrm{~nm}$ indium tin oxide (ITO). It supports guided transversal magnetic (TM) modes in the entire spectral region of interest. The lattice constant $a$ of the wire pairs is varied within the set of samples from $a=400 \mathrm{~nm}$ to $a=1050 \mathrm{~nm}$ in steps of $25 \mathrm{~nm}$. All other parameters are kept constant. As a control experiment, we have also investigated a second set of arrays of wire pairs (control arrays) deposited directly on top of a substrate (quartz glass covered with $5 \mathrm{~nm}$ ITO), i.e., without a $\mathrm{HfO}_{2}$ waveguide. Here no guided modes are supported.

Both sets of samples have been fabricated by electronbeam lithography and a lift-off process. The individual arrays have a footprint of $100 \mu \mathrm{m} \times 100 \mu \mathrm{m}$. All layers (ITO, $\mathrm{HfO}_{2}$, gold, and $\mathrm{MgF}_{2}$ ) have been evaporated by electron-beam evaporation in a high-vacuum chamber at pressures below $10^{-6}$ mbar. A top-view electron micrograph of a magnetic photonic crystal with $a=800 \mathrm{~nm}$ is depicted in Fig. 1(c). The transmittance properties of the magnetic photonic crystals and the corresponding control samples are studied with a homebuilt white-light setup for normal incidence and for TM polarization of the incident light [see Fig. 1(b)]. Normalization of the spectra is accomplished with respect to the transmittance of the bare waveguide and the bare substrate, respectively. Since the transmittance properties critically depend on the angle of incidence, great care has been taken to orient the surface of the samples normal to the incident light beam. Furthermore, the effective full opening angle of the incident light beam is reduced to less than $1.5^{\circ}$.

Figure 2(a) shows measured transmittance spectra for two selected samples, corresponding to the sample and measurement geometry depicted in Fig. 1(b). Figure 3(a) gives an overview on the complete set of 27 samples with different lattice constants $a$, depicted on an intuitive gray scale (black corresponds to zero transmittance, white to near unity transmittance). For $a=400 \mathrm{~nm}$, the magnetic resonance of the wire pairs corresponds to the dip in transmittance centered at $\lambda=1200 \mathrm{~nm}$ wavelength. The periodic arrangement of wire pairs allows the excitation of a quasiguided TM-waveguide mode even for normal incidence. The corresponding Bragg resonance coincides with the electric resonance of the wire pairs for small $a$. An avoided crossing of the latter two modes [11] leads to two new resonances at $\lambda=630 \mathrm{~nm}$ and $\lambda=780 \mathrm{~nm}$ wavelength, respectively. By increasing the lattice constant $a$, the Bragg resonance shifts to a longer wavelength (see dotted red curve) while the magnetic resonance initially $(a<650 \mathrm{~nm}$ ) keeps its spectral position (see dotted yellow curve). Small variations of the spectral position of the

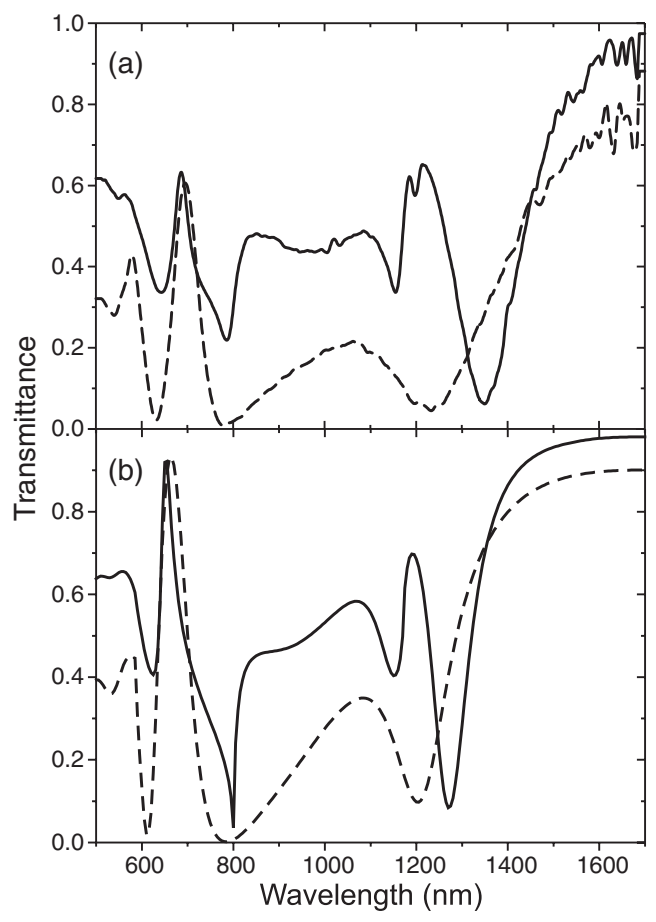

FIG. 2. (a) Experimental transmittance spectra for the geometry described in Fig. 1(b) for $a=400 \mathrm{~nm}$ (dashed line) and $a=$ $800 \mathrm{~nm}$ (solid line), respectively. (b) Corresponding calculated spectra. 

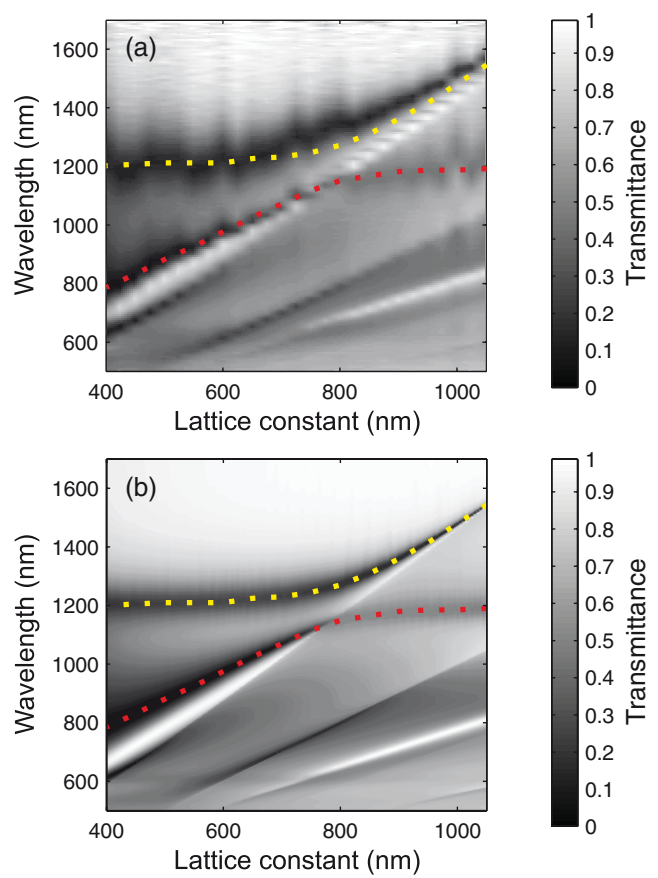

FIG. 3 (color). (a) Measured and (b) calculated transmittance on an intuitive gray scale versus lattice constant $a$ and wavelength $\lambda$ for the magnetic photonic crystals. In the experiment, the data from 27 different samples are summarized [two examples are shown in Fig. 2(a)]. The dotted red and yellow curves are guides to the eye.

magnetic resonance can be attributed to variations of $w$ for the different arrays due to fabrication tolerances. For $a \approx$ $800 \mathrm{~nm}$, we observe an avoided crossing of the Bragg resonance and the magnetic resonance. It results from the strong coupling of the periodic arrangement of magnetic dipoles provided by the wire pairs to the quasiguided waveguide mode. Further increasing of the lattice constants $(a>900 \mathrm{~nm})$ spectrally detunes the Bragg and the magnetic resonance, which basically yields an uncoupled system again. The other spectral features which shift to a larger wavelength with increasing lattice constant correspond to Rayleigh anomalies (for details see below) or to higher-order waveguide modes.

Our experimental findings concerning the avoided crossing of the Bragg and the magnetic resonance are also validated by rigorous numerical calculations based on a scattering-matrix approach [16]. The parameters in our calculations are permittivity of the ITO film $\epsilon_{\text {ITO }}=3.61$, permittivity of the $\mathrm{HfO}_{2}$ waveguide $\epsilon_{\mathrm{HfO}_{2}}=3.8$, permittivity of the glass substrate $\epsilon_{\text {glass }}=2.14$, and permittivity of the $\mathrm{MgF}_{2}$ layer $\epsilon_{\mathrm{MgF}_{2}}=1.9$. Values for the permittivity of gold are taken from Ref. [17]. The geometrical parameters correspond to Fig. 1(b). The calculated transmittance spectra are shown in Fig. 3(b). Here the same normalization and graphical representation is utilized as for the experimental data. A direct comparison between experimental and calculated spectra reveals excellent agreement. In particular, the spectral position and the width of the avoided crossing are accurately reproduced by the numerical calculations. The calculated field distributions of several relevant modes are depicted in the supporting online material [18]).

The second set of samples [control arrays, i.e., no $\mathrm{HfO}_{2}$ waveguide in Fig. 1(b)] corresponds to the case of weakly coupled magnetic atoms. Because of the absence of the waveguide, the reference samples do not exhibit a Bragg resonance. Instead, the reference samples exhibit a different scenario for the dependence of the transmittance spectra on $a$. Whenever an integer multiple of the wavelength of light in the substrate or vacuum becomes smaller than the actual lattice constant, a Rayleigh anomaly occurs [19]. These Rayleigh anomalies are connected with the appearance of new grating orders and can have a strong influence on the line shape of other resonances, e.g., electric-dipole resonances [20]. However, an avoided crossing with the magnetic resonance is not expected because the damping is larger than the anticipated splitting energy of the weakly coupled system.

The measured transmittance spectra for the control arrays are depicted in Fig. 4(a). The graphical presentation and the normalization is according to Fig. 3(a). For the control samples, the magnetic resonance is shifted by approximately $200 \mathrm{~nm}$ to a shorter wavelength compared to the magnetic photonic crystals. This spectral shift results from a variation of the dielectric environment (no $\mathrm{HfO}_{2}$ waveguide) and a slightly smaller width $w=200 \mathrm{~nm}$ of the wire pairs. The first Rayleigh anomaly corresponds to the opening of a new diffraction order in the substrate. As
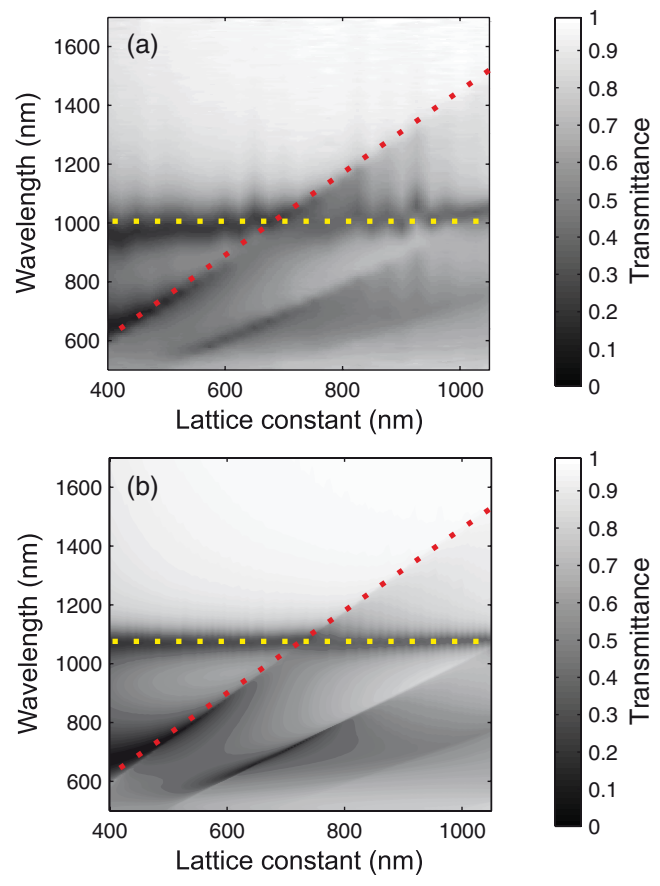

FIG. 4 (color). (a) Measured and (b) calculated transmittance on an intuitive gray scale versus lattice constant $a$ and wavelength $\lambda$ for the control samples (no $\mathrm{HfO}_{2}$ waveguide). The dotted red and yellow curves are guides to the eye. 
expected for the situation of weak coupling, this Rayleigh anomaly simply crosses the magnetic resonance when the lattice constant $a$ is increased [see dotted red and yellow curves in Fig. 4(a)]. This observation is also reproduced by the numerical calculation [see Fig. 4(b)]. Note that the first order Rayleigh anomaly for vacuum can also be observed in the case of the magnetic photonic crystals (see Fig. 3). However, the coresponding Rayleigh anomaly for the substrate is absent due to total internal reflection at the waveguide-substrate interface.

Before concluding, it is also interesting to look at Figs. 3 and 4 from the viewpoint of two notions: "photonic crystal" on the one hand and "photonic metamaterial" on the other hand. Both expressions have frequently been used in the literature (not quite consistently though). What is the difference between the two? Remarkably, closely similar building blocks (magnetic atoms) are used in Figs. 3 and 4 (also see Fig. 1). Their spacing (lattice constant) is similar as well. Nevertheless, the optical properties are clearly rather different: In one case, one gets a pronounced avoided crossing (Fig. 3). In sharp contrast, the lattice constant $a$ has no major influence on the magnetic resonance in the other case (Fig. 4). This qualitative difference arises from the fact that coupling between the different building blocks (magnetic atoms) via the electromagnetic field is strong in Fig. 3-due to the presence of a slab waveguide-whereas coupling is of minor importance without the slab waveguide (Fig. 4). Thus, the latter type of structure can be well described as an effective medium (a "metamaterial"), whereas the other structure (Fig. 3) reveals pronounced signatures of Bloch waves (Bragg scattering), commonly ascribed to photonic crystals.

In conclusion, we have experimentally and theoretically investigated periodic arrays of gold-wire pairs on top of a dielectric slab waveguide. These structures serve as a simple model system for one-dimensional magnetic photonic crystals. We observe an avoided crossing of the magnetic resonance of the wire pairs and the Bragg resonance. A compound mode results from strong coupling of the periodic arrangement of magnetic dipoles provided by the wire pairs to the waveguide mode. In contrast, for weakly coupled magnetic atoms, i.e., for wire pairs on top of a substrate which does not support guided modes, the avoided crossing and, hence, the strong coupling of magnetic dipoles and photonic modes is absent. The experimental results are accurately reproduced by numerical calculations based on a scattering-matrix approach. These investigations raise hopes that photonic crystals composed of artificial magnetic atoms might open a whole new world of opportunities predicted by theory. For example [21], complete 3D photonic band gaps can arise from 1D magnetic or dielectric layer stacks. The fabricational challenges, however, are still considerable as such effective 1D structures are connected with a 3D periodic substructure of artificial atoms.

We thank C. M. Soukoulis and C. Enkrich for stimulating discussions in the early phase of this work. We ac- knowledge support by the Deutsche Forschungsgemeinschaft (DFG) and the State of Baden-Württemberg through the DFG-Center for Functional Nanostructures (CFN) within subproject A1.5. The research of M.W. is further supported by Project No. DFG-We 1497/9-1 and that of S. L. through "Helmholtz-Hochschul-Nachwuchsgruppe" (VH-NG-232).

[1] A. Pimenov, A. Loidl, P. Przyslupski, and B. Dabrowski, Phys. Rev. Lett. 95, 247009 (2005).

[2] J. D. Joannopoulos Photonic Crystals: Molding the Flow of Light (Princeton University Press, Princeton, NJ, 1995).

[3] J. B. Pendry, A. J. Holden, D. J. Robbins, and W. J. Stewart, IEEE Trans. Microwave Theory Tech. 47, 2075 (1999).

[4] S. Linden, C. Enkrich, M. Wegener, J. F. Zhou, T. Koschny, and C. M. Soukoulis, Science 306, 1351 (2004).

[5] S. Zhang, W. Fan, N.C. Panoiu, K. M. Malloy, R. M. Osgood, and S. R. J. Brueck, Phys. Rev. Lett. 95, 137404 (2005).

[6] A. N. Grigorenko, A. K. Geim, H. F. Gleeson, Y. Zhang, A. A. Firsov, I. Y. Khrushev, and J.Petrovic, Nature (London) 438, 335 (2005).

[7] G. Dolling, C. Enkrich, M. Wegener, J.F. Zhou, C. M. Soukoulis, and S. Linden, Opt. Lett. 30, 3198 (2005).

[8] V. M. Shalaev, W. S. Cai, U. K. Chettiar, H. K. Yuan, A. K. Sarychev, V. P. Drachev, and A. V. Kildishev, Opt. Lett. 30, 3356 (2005).

[9] C. Enkrich, M. Wegener, S. Linden, S. Burger, L. Zschiedrich, F. Schmidt, J.F. Zhou, T. Koschny, and C. M. Soukoulis, Phys. Rev. Lett. 95, 203901 (2005).

[10] K. C. Huang, P. Bienstman, J. D. Joannopoulos, K. A. Nelson, and S. Fan, Phys. Rev. Lett. 90, 196402 (2003).

[11] A. Christ, S. G. Tikhodeev, N. A. Gippius, J. Kuhl, and H. Giessen, Phys. Rev. Lett. 91, 183901 (2003).

[12] M. W. Klein, T. Tritschler, M. Wegener, and S. Linden, Phys. Rev. B 72, 115113 (2005).

[13] V.N. Astratov, D. M. Whittaker, I. S. Culshaw, R. M. Stevenson, M.S. Skolnick, T. F. Krauss, and R. M. De La Rue, Phys. Rev. B 60, R16 255 (1999).

[14] A. N. Lagarkov and A. K. Sarychev, Phys. Rev. B 53, 6318 (1996).

[15] J. B. Pendry, A. J. Holden, W. J. Stewart, and I. Youngs, Phys. Rev. Lett. 76, 4773 (1996).

[16] D. M. Whittaker and I. S. Culshaw, Phys. Rev. B 60, 2610 (1999).

[17] P. B. Johnson and R. W. Christy, Phys. Rev. B 6, 4370 (1972).

[18] See EPAPS document No. E-PRLTAO-97-052634 for snapshots of the electric- and magnetic-field distributions for several relevant modes discussed in the Letter. For more information on EPAPS, see http://www.aip.org/ pubservs/epaps.html.

[19] A. Hessel and A. A. Oliner, Appl. Opt. 4, 1275 (1965).

[20] B. Lamprecht, G. Schider, R. T. Lechner, H. Ditlbacher, J. R. Krenn, A. Leitner, and F. R. Aussenegg, Phys. Rev. Lett. 84, 4721 (2000).

[21] I. V. Shadrivov, A. A. Sukhorukov, and Y. S. Kivshar, Phys. Rev. Lett. 95, 193903 (2005). 